\title{
Correction to: Multiple Score Comparison: a network meta-analysis approach to comparison and external validation of prognostic scores
}

Sarah R. Haile', Beniamino Guerra', Joan B. Soriano ${ }^{2}$ and Milo A. Puhan ${ }^{1,3^{*}}$

\section{Correction}

Following publication of the original article [1], a member of the writing group reported that his name is misspelt. The paper should appear in Pubmed under "Ter Riet G", not as "Riet GT".

\section{Author details}

${ }^{1}$ Epidemiology, Biostatistics and Prevention Institute, University of Zurich, Zurich, Switzerland. ${ }^{2}$ Servicio de Neumología, Instituto de Investigación del Hospital Universitario de la Princesa (IISP), Universidad Autónoma de Madrid, Madrid, Spain. ${ }^{3}$ Epidemiology \& Department of Epidemiology, Johns Hopkins Bloomberg School of Public Health, Baltimore, USA.

Received: 5 February 2018 Accepted: 5 February 2018

Published online: 12 February 2018

\section{Reference}

1. Haile SR, Guerra B, Soriano JB, Puhan MA. Multiple Score Comparison: a network meta-analysis approach to comparison and external validation of prognostic scores. BMC Med Res Methodol. 2017;17:172. https://doi.org/10.1186/s12874-017-0433-2.

* Correspondence: miloalan.puhan@uzh.ch

'Epidemiology, Biostatistics and Prevention Institute, University of Zurich, Zurich, Switzerland

${ }^{3}$ Epidemiology \& Department of Epidemiology, Johns Hopkins Bloomberg School of Public Health, Baltimore, USA 